\title{
DEFORMATIONS OF FINITE DIMENSIONAL ALGEBRAS AND THEIR IDEMPOTENTS
}

\author{
M. SCHAPS
}

\begin{abstract}
Let $B$ be a finite dimensional algebra over an algebraically closed field $K$. If we represent primitive idempotents by points and basis vectors in $e_{i} B e_{j}$ by "arrows" from $e_{j}$ to $e_{i}$, then any specialization of the algebra acts on this directed graph by coalescing points. This implies that the number of irreducible components in the scheme parametrizing $n$-dimensional algebras is no less than the number of loopless directed graphs with a total of $n$ vertices and arrows. We also show that the condition of having a distributive ideal lattice is open.
\end{abstract}

1. Introduction. In [5], P. Gabriel leaves as an open challenge the determination of the number of irreducible components in the structure constant scheme $\operatorname{Alg}_{n}$, which parametrizes multiplication structures of $n$-dimensional algebras. The number of quivers in which each vertex is either a source or a sink provides a very weak lower bound, since the radical-squared zero algebras generated by such quivers are known to be rigid. This was converted into a numerical bound by Mazzola in [11]. However, as an examination of the list of irreducible components for dimension 5 will show, most of the quivers of rigid radical-squared zero algebras have vertices which are neither sources nor sinks. Let the directed graph described in the abstract above be called the basis graph of an algebra, and conversely, given a directed graph, let the unique radical-squared zero algebra with that configuration of idempotents and radical elements be called the basis graph algebra generated by that graph. The long range purpose of the present work is to develop machinery for determining and classifying the irreducible components of $\mathrm{Alg}_{n}$ by studying the effect of deformation on the basis graph, but one of its immediate applications is to prove that every irreducible component contains no more than one loopless basis graph algebra. For example, in dimension 5 there are nine loopless basis graph algebras, contained in nine of the ten different irreducible components. Thus in low dimensions the number of loopless basis graphs gives a fairly reasonable lower bound.

A different strand in the analysis of $\mathrm{Alg}_{n}$ began with the work of $\mathrm{M}$. Gerstenhaber on formal deformations of algebras [6] and culminated in the work of Flanigan $[\mathbf{3}, \mathbf{4}]$. In the second section we broaden Flanigan's "straightening-out" theorems, [3], so that they will be valid for deformations over an arbitrary base space. In the third section we stratify $\operatorname{Alg}_{n}$ by associating to each algebra a weighted basis graph, demonstrate that this is indeed an algebraic stratification, and show that the only

Received by the editors May 22, 1986 and, in revised form, May 7, 1987. Presented at Representations of Finite Dimensional Algebras, Mathematisches Forschungsinstitut, Oberwolfach, March 26, 1986.

1980 Mathematics Subject Classification (1985 Revision). Primary 16A46, 16A58. 
possible specializations are obtained by holding the vertices fixed and increasing the weights, or by coalescing vertices and adding loops. This gives the desired lower bound.

In $\S 4$ we concentrate on algebras which are candidates to be of finite representation type, and prove the openness of the distributive ideal lattice condition. The chart for the 2- and 3-arrow algebras with distributive ideal lattice in dimension 6 is included as an illustration.

2. The "straightening-out" theorems. Let $K$ be an algebraically closed field. If $A(t)$ is a finite dimensional $K$-algebra whose structure constants are formal power series in an indeterminate $t$, then any set of idempotents and matrix units for the special algebra $A(0)$ lifts to a corresponding set for $A(t)$. However, since the radical of $A(0)$ may be larger than the radical of $A(t)$, idempotents which were primitive in $A(0)$ may no longer be primitive in $A(t)$ (see [3]).

When we pass to deformations over an arbitrary base space, and in particular, over an arbitrary component of $\mathrm{Alg}_{n}$, we have to exercise more care. Central idempotents in a closed fiber always determine a unique etale neighborhood of the base space whose fibers are central idempotents. This fact is frequently quoted to show that the number of irreducible blocks in an algebra is a lower semicontinuous function, i.e., it cannot increase when we pass to some specialization of an algebra. However, noncentral idempotents do not lift uniquely in a deformation over an algebraic base space. As a result, liftability of idempotents was not used as one of the criteria to determine possible deformations in the studies of deformations of 5-dimensional algebras $[\mathbf{7}, \mathbf{1 1}]$.

The extra ingredient needed to extend the formal results of the algebraic situation is the Artin approximation theorem from the theory of etale coverings. This will allow us to make a change of parameter space, after which the idempotents and matrix units can be lifted as desired. We will need several concepts from algebraic geometry: morphisms of finite type, etale morphisms and etale neighborhoods. For the convenience of the reader we have collected the definitions in an appendix at the back of the paper. For those interested in gaining more fluency in the applications of algebraic geometry to algebra, we have included references to the various results needed during the course of the proof.

There are several competing definitions of deformation in the context of finite dimensional algebras. We will use the following:

DEFINITION. Let $\left(\mathscr{C}, t_{0}\right)$ be any pointed affine scheme, with $\mathscr{C}=\operatorname{Spec}(R)$ for $R$ a commutative affine ring over $K$ and $t_{0}$ a closed point corresponding to a maximal ideal $m_{0}$ of $R$. A flat deformation $B$ of $B_{0}$ over $\left(\mathscr{C}, t_{0}\right)$ is a flat $R$-algebra $B$ together with an isomorphism of $B_{0}$ with $B \otimes_{R} R / m_{0}$.

REMARK. In more classical terminology, when the fibers over general closed points of $\mathscr{C}$ are all isomorphic to an algebra $A$, then $A$ is also called a deformation of $B$, and the relationship is denoted by $A \rightarrow B$. This can also be read as " $A$ specializes to $B . "$

Over an algebraically closed field, the Wedderburn structure theorems have a particularly simple form: any finite dimensional algebra is a split extension of its nilpotent radical $J$ by a subalgebra $S$ isomorphic to sum of matrix blocks $M_{n}(K)$. For each such block one can choose a basis of matrix units $E_{i j}$ with the standard multiplication $E_{i j} \cdot E_{j k}=E_{i k}$. 
Let $B$ be a matrix block isomorphic to $M_{n}(K)$. There are various ways of choosing subalgebras of $B$ which are also direct sums of matrix blocks, and two of these are of particular importance in deformation theory. One way is to choose a partition $n=n_{1}+\cdots+n_{r}$ and consider the subalgebra generated by the matrix units in the diagonal blocks of these dimensions. The other way only works if $n$ has a nontrivial decomposition as a product $n=c \cdot d$ of two integers. In this case $M_{n}(K) \stackrel{\sim}{\rightarrow} M_{c}\left(M_{d}(K)\right)$, and the matrix units of the outer matrix representations are each sums of $d$ of the original matrix units. In this second case the idempotents are not primitive. Each idempotent is the sum of $d$ of the original idempotents.

DEFINITION. Let $B$ be an algebra which is a finitely generated free module over a commutative ring $R$. A matrix decomposition set $e$ for $B$ will be a set of matrix units for an $R$-subalgebra of $B$ isomorphic to $\bigoplus_{i=1}^{m} M_{r_{i}}(R)$. If the sum of the idempotents is 1 , we will call $e$ a complete matrix decomposition set. If the idempotents are primitive we will call $e$ a primitive matrix decomposition set, and if $e$ spans $B / \operatorname{Rad} B$ we will call $e$ a Wedderburn set for $B$.

Proposition 1 ("Straightening-OUt ThEOREM"). Let $B$ be a flat deformation of a finite dimensional algebra $B_{0}$ over a pointed affine scheme $\left(\mathscr{C}, t_{0}\right)$, with $\mathscr{C}=\operatorname{Spec}(R)$. Let $e_{0}$ be a complete matrix decomposition set for $B_{0}$ over $K$. Then there is an etale neighborhood $\left(\mathscr{C}^{\prime}, t_{0}^{\prime}\right)$ of $\left(\mathscr{C}, t_{0}\right)$, with $\mathscr{C}^{\prime}=\operatorname{Spec}\left(R^{\prime}\right)$, such that $e_{0}$ lifts to a complete matrix decomposition set for the $R^{\prime}$ algebra $B \otimes_{R} R^{\prime}$.

PROOF. Any firitely generated flat module over a local Noetherian ring is free [8, III, 9.1A(f)] so if we denote the (Zariski) localization of $R$ at $m_{0}$ by $R_{0}$, then $B \otimes_{R} R_{0}$ is a free $R_{0}$-module. Thus replacing $\mathscr{C}$ by an open neighborhood of $t_{0}$, we may assume that $B$ is free as an $R$-module.

Let $\widehat{R}_{0}$ denote the completion of $R$ at $t_{0}$, with $\widehat{\mathscr{C}}_{0}=\operatorname{Spec}\left(\widehat{R}_{0}\right)$, and let $m_{0}$ be the unique maximal ideal of $\widehat{R}_{0}$. Let $f_{1}, \ldots, f_{r}$ be the idempotents in the complete matrix decomposition set $e_{0}$. Since idempotents can always be lifted modulo a nilpotent ideal, they can be lifted inductively from $\widehat{R}_{0} / m_{0}^{k}$ to $\widehat{R}_{0} / m_{0}^{k+1}$ for $k=$ $1,2, \ldots$, so they can in fact be lifted to the completion. Once lifted, they can be adjusted to be orthogonal $\left[10\right.$, p. 53]. It suffices, in fact, to lift $f_{1}, \ldots, f_{r-1}$ to an orthogonal set of idempotents $\hat{f}_{1}, \ldots, \hat{f}_{r-1}$, for then we can define

$$
\hat{f}_{r}=1-\left(\hat{f}_{1}+\cdots+\hat{f}_{r-1}\right)
$$

and the resulting set of idempotents $\hat{f}_{1}, \ldots, \hat{f}_{r}$ will be complete and orthogonal.

Choose an $R$-module basis $v_{0}, \ldots, v_{n-1}$ for $B$ over $R$, such that $v_{0}$ is the identity of the given algebra structure on $B$. Let $\alpha=\left(a_{i j}^{k}\right) \in R^{3 n}$ be the set of $R$-coordinates of the structure constant tensor of the multiplication in $B$ with respect to the basis $v_{0}, \ldots, v_{n-1}$, i.e.

$$
v_{i} \cdot \alpha v_{j}=\sum a_{i j}^{k} v_{k} .
$$

(The tensor $\alpha$ determines a morphism from $\mathscr{C}=\operatorname{Spec}(R)$ into the scheme of structure constant tensors $\mathrm{Alg}_{n}$. Thus in what follows one could replace $\mathscr{C}$ by $\mathrm{Alg}_{n}$ and afterwards define $\mathscr{C}^{\prime}=\mathscr{C} \times \operatorname{Alg}_{n}\left(\operatorname{Alg}_{n}\right)^{\prime}$.)

Let $W \subset \mathscr{C} \times V^{r}$ be the algebraic scheme determined by the $\alpha$-dependent equations $f_{i} \cdot \alpha f_{j}=\delta_{i j} f_{j}, f_{1}+\cdots+f_{r}=1$, which are quadratic equations in the coordinates of the $f_{i}$ and $f_{j}$, together with $n$ linear equations. Each closed fiber 
determined by these equations is embedded in an affine space of dimension $n r$, and thus the projection $\pi: W \rightarrow \mathscr{C}$ is a morphism of finite type. The set $\hat{f}_{1}, \ldots, \hat{f}_{r}$ gives a section of $\hat{\mathscr{C}}_{0}$ into the base extension $W \times \hat{\mathscr{C}}_{0}$, so by Artin's approximation theorem [1], there exists an etale morphism $p: \mathscr{C}^{\prime} \rightarrow \mathscr{C}$ whose image contains $t$, such that there is a section $\sigma: \mathscr{C}^{\prime} \rightarrow W \times \mathscr{f} \mathscr{C}^{\prime}$ in which the image of $t_{0}$ is $f_{1}, \ldots, f_{r}$. (Equivalently, as suggested by the referee, one can use the formal criterion for smoothness to show that $\pi$ is smooth and thus $\mathscr{C}^{\prime}$ and $\sigma$ exist as asserted.) Let $\bar{f}_{1}, \ldots, \bar{f}_{r}$ be the corresponding set of idempotents in $\mathscr{C}^{\prime} \times V^{r}$. We thus have a complete orthogonal idempotent set for $B$.

Once a lifting has been chosen for the idempotents, there is a standard technique for lifting the matrix units. One lifts each $E_{1 s}, s>1$, and projects onto the proper Peirce component by $\bar{E}_{11}$ and $\bar{E}_{s s}$. Discarding from $\mathscr{C}^{\prime}$ the closed subsets on which right multiplication by $E_{1 s}$ is not an isomorphism from $\bar{E}_{s s} B^{\prime} \bar{E}_{11}$ to $\bar{E}_{s s} B^{\prime} \bar{E}_{s s}$, we let $\bar{E}_{s 1}$ be the inverse image of $\bar{E}_{s s}$ under this isomorphism, so that $\bar{E}_{s 1} \cdot \bar{E}_{1 s}=\bar{E}_{s s}$. Multiplying this equation on the left by $\bar{E}_{s 1}$, we find that

$$
\bar{E}_{1 s} \cdot \bar{E}_{s 1} \cdot \bar{E}_{1 s}=\bar{E}_{1 s} \cdot \bar{E}_{s s}=\bar{E}_{11} \cdot \bar{E}_{1 s} .
$$

Again discarding the closed set on which right multiplication by $\bar{E}_{1 s}$ is not an isomorphism from $\bar{E}_{11} B^{\prime} \bar{E}_{11}$ to $\bar{E}_{11} B^{\prime} \bar{E}_{s s}$, we find that

$$
\bar{E}_{1 s} \cdot \bar{E}_{s 1}=\bar{E}_{11} \text {. }
$$

Thus $p:\left(\mathscr{C}^{\prime}, s_{0}\right) \rightarrow\left(\mathscr{C}, t_{0}\right)$ is the desired etale neighborhood.

DEFINITION. Let $e$ be a matrix decomposition set for a free $R$-algebra $B$. We will say that an $R$-module basis $x$ of $B$ respects $e$ if it contains $e$, and multiplication of a basis element by an element of $e$ gives 0 or another basis element. If $J$ is a two-sided ideal which is a flat $R$-module, then we will say that $x$ respects $J$ if some subset forms a basis for $J$.

COROLlaRY 1.1. Suppose $B^{\prime}$ is a deformation of $B_{0}$ over $R^{\prime}$, as in the theorem, and $e$ is some lifting of a matrix decomposition set of $B_{0}$ to $B^{\prime}$. Let $J_{1} \supset J_{2} \supset$ .. $\supset J_{l}$ be a nested sequence of flat ideals. If $x_{0}$ is a basis of $B_{0}$ which respects $e_{0}$ and the ideals $J_{i} \otimes_{R} R / m_{0}$, then over an open Zariski neighborhood $\left(\mathscr{C}^{\prime \prime}, t_{0}\right)$ of $\left(\mathscr{C}, t_{0}\right)$ with $\mathscr{C}^{\prime \prime}=\operatorname{Spec}\left(R^{\prime \prime}\right)$, we can find a basis $x$ of $B^{\prime \prime}=B^{\prime} \otimes_{R^{\prime}} R^{\prime \prime}$ which respects $e$ and the ideals $J_{i}$.

PROOF. We proceed by reverse induction on the index $l$ of $J_{l}$. Restricting if necessary to a Zariski open neighborhood of $t_{0}$, we choose any basis for $J_{l}$ which is linearly independent over $t_{0}$, and perform a $K$-linear transformation so that it coincides with a subbasis of $x_{0}$ over $t_{0}$. We then take this transformed basis as part of a local basis for $J_{l-1}$, and transform as necessary. After performing this operation for all $i$, we lift the elements of $e_{0}$ by $e$. If any elements of $x_{0}$ have not yet been lifted, we take arbitrary liftings, and restrict to the Zariski open set on which they are linearly independent.

Since $x_{0}$ respects $e_{0}$, each basis element lies in some factor $e_{j} B_{0} e_{i}$ of a Peirce decomposition, where $e_{i}, e_{j}$ are idempotents from $e_{0}$. If $\bar{x}_{l}$ is a lifted basis element, we replace it by $\tilde{e}_{j} \bar{x}_{l} \tilde{e}_{i}$. Within any set of matrix units $\left(e_{i j}\right)$, we choose one idempotent, say $e_{11}$, and obtain a basis of $\tilde{e}_{j j} B \tilde{e}_{k k}$ by multiplying a basis of $\tilde{e}_{11} B \tilde{e}_{11}$ by matrix units $\tilde{e}_{j 1}$ and $\tilde{e}_{1 k}$. The resulting basis $\tilde{x}$ has the required closure property under multiplication by elements of $e$. Q.E.D. 
DEfinition. A $K$-algebra $A$ is sober if $A / \operatorname{Rad} A$ is a product of fields.

To any algebra $A$ we can associate a sober algebra $A^{-}$unique up to isomorphism.

DEFINITION. Let $A$ be an algebra, and let $e$ be a Wedderburn set for $A$, i.e. a complete primitive matrix decomposition set which spans $A / \operatorname{Rad} A$. Let $f_{1}, \ldots, f_{r}$ be a subset of $e$ formed by choosing one idempotent for each matrix block, and set $f=f_{1}+\cdots+f_{r}$, which is also an idempotent of $A$. Then the algebra $A^{-}=f A f$ is called the skeleton of $A$ (as in Flanigan [3]).

REMARK. Up to isomorphism, $A^{-}$is independent of the choice of idempotent set and of the choice of $f_{1}, \ldots, f_{r}$, since for any other choice there is an automorphism of $A$ carrying one into the other. $A^{-}$is sober because

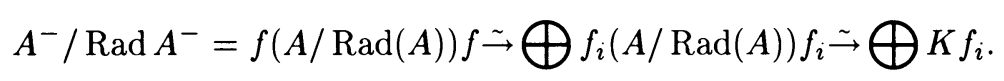

Algebras with the same skeleton are called Morita equivalent. It is well known that the representation theory of an algebra depends only on its Morita equivalence class, and thus representation theory can be done with sober algebras. We claim that essentially the same is true for deformation theory - the deformations of an algebra depend only on its Morita equivalence class. The isomorphism classes of algebras appearing in deformations of an algebra $B_{0}$ are in one-to-one correspondence with algebras appearing in deformations of its skeleton $B_{0}^{-}$.

COROLlaRY 1.2. If $B$ is a deformation of $B_{0}$ over $\left(\mathscr{C}, t_{0}\right)$, then there is an etale morphism $p:\left(\mathscr{C}^{\prime}, t_{0}^{\prime}\right) \rightarrow\left(\mathscr{C}, t_{0}\right)$ and a deformation $\bar{B}$ of $B_{0}^{-}$over $\left(\mathscr{C}^{\prime}, t_{0}^{\prime}\right)$ such that $B \times \mathscr{C}^{\prime}$ has closed fibers which are Morita equivalent to the corresponding fibers of $\bar{B}$.

PROOF. Let $e_{0}$ be a complete primitive matrix decomposition set for $B_{0}$, and $x_{0}$ a basis for $B_{0}$ which respects it. By Corollary 1.1 there is an etale morphism $p:\left(\mathscr{C}^{\prime}, s_{0}\right) \rightarrow\left(\mathscr{C}, t_{0}\right)$ over which $e_{0}$ and $x_{0}$ lift to $e$ and $x$ in $B^{\prime}=B \times \mathscr{C} \mathscr{C}^{\prime}$. Let $f_{1}, \ldots, f_{r}$ be a subset of the idempotents in $e_{0}$, chosen one from each matrix block, and let $f_{0}=f_{1}+\cdots+f_{r}$. Let $f_{1}^{\prime}, \ldots, f_{r}^{\prime}$ be the corresponding idempotent sections in $B^{\prime}$, and set $f^{\prime}=f_{1}^{\prime}+\cdots+f_{r}^{\prime}$. The skeleton $B_{0}^{-}=f_{0} B_{0} f_{0}$ is spanned by all elements in the basis $x_{0}$ lying in Peirce blocks of the form $f_{i} B_{0} f_{j}$, and the subalgebra $\bar{B}=f^{\prime} B^{\prime} f^{\prime}$ of $B^{\prime}$ is spanned by the corresponding elements of the $R^{\prime}$ module basis $x$ of $B^{\prime}$. Thus $\bar{B}$ is a free $R^{\prime}$ module over $\mathscr{C}^{\prime}$ whose closed fiber at $s_{0}$ is $B_{0}^{-}$. It is therefore a deformation of $B_{0}^{-}$.

Let $A$ be any closed fiber of $B^{\prime}$, let $f^{\prime \prime}=f_{1}^{\prime \prime}+\cdots+f_{r}^{\prime \prime}$ be the restriction of the idempotent $f^{\prime}$ to this closed fiber, and let $\bar{A}=f^{\prime \prime} A f^{\prime \prime}$ be the closed fiber of the deformation $\bar{B}$ of $B_{0}^{-}$. We have to show that $A$ and $\bar{A}$ are Morita equivalent.

For each $i$, choose a Wedderburn set $\bar{e}_{i}$ for $\bar{A}_{i i}=f_{i}^{\prime \prime} \bar{A} f_{i}^{\prime \prime}=f_{i}^{\prime \prime} A f_{i}^{\prime \prime}$. The subalgebra of $\bar{A}_{i i}$ generated by $\bar{e}_{i}$ will have the form $\bigoplus M_{c_{i j}}(K)$. In fact, the union $\bar{e}$ of the $\bar{e}_{i}$ will be a complete primitive matrix decomposition set for $\bar{A}$. In $A$, each $f_{i}$ is an idempotent in a $d_{i} \times d_{i}$ block of matrix units. For each $i$, we multiply each primitive matrix decomposition set $\bar{e}_{i}$ by the matrix units corresponding to $f_{i}$ and in this way we produce a complete matrix decomposition set $e^{\prime}$ for $A$, generating a subalgebra of the form

$$
\bigoplus_{i} \bigoplus_{j} M_{d_{i}}\left(M_{c_{i j}}(K)\right) \stackrel{\sim}{\rightarrow} \bigoplus_{i} \bigoplus_{j} M_{d_{i} c_{i j}}(K)
$$


Now decompose $A / \operatorname{Rad} A$ into a sum of matrix blocks $\bigoplus M_{n_{l}}(K)$. Any matrix subalgebra of $A$, being irreducible, must be entirely contained in one of the $n_{l} \times n_{l}$ blocks, and this defines a function $\phi(i, j)=l$ such that

$$
\bigoplus_{\phi(i, j)=l} M_{d_{i} c_{i j}}(K) \subset M_{n_{l}}(K), \quad \text { with } n_{l}=\sum_{\phi(i, j)=l} d_{i} c_{i j}
$$

Since $e^{\prime}$ is a complete matrix decomposition set, it generates a complete set of diagonal blocks within $A / \operatorname{Rad} A$, and can therefore be extended to a Wedderburn set $w$ for $A$.

By construction, all of the matrix decomposition sets $\bar{e}, e^{\prime}$, and $w$ respect the set of idempotents $\left(f_{1}^{\prime \prime}, \ldots, f_{r}^{\prime \prime}\right)$. Thus $\bar{A} / \operatorname{Rad}(\bar{A})=f^{\prime \prime}(A / \operatorname{Rad}(A)) f^{\prime \prime}$ will be spanned by all the elements of $w$ lying in the subset $f^{\prime \prime} w f^{\prime \prime}$, and will have a matrix decomposition

$$
\bar{A} / \operatorname{Rad}(\bar{A}) \stackrel{\sim}{\rightarrow} \bigoplus M_{m_{l}}(K), \quad \text { with } m_{l}=\sum_{\phi(i, j)=l} c_{i j}
$$

This shows that $f^{\prime \prime} w f^{\prime \prime}$ is a Wedderburn set for $\bar{A}$. Its idempotents coincide with the idempotents in $\bar{e}$.

For each $l$, we let $(i, j)$ be the first pair in lexicographical order with $\phi(i, j)=l$, and let $g_{i}$ be the first idempotent in $f_{i}^{\prime \prime} M_{d_{i}}\left(M_{c_{i j}}(K)\right) f_{i}^{\prime \prime} \sim M_{c_{i j}}(K)$.

Let $g=\sum g_{l}$. Then $g A g=g \bar{A} g$ is the common skeleton of $A$ and $\bar{A}$. This proves that $A$ and $\bar{A}$ are Morita equivalent.

REMARK. Any deformation of a nonsober algebra $B_{0}$ is obtained from a deformation of its skeleton by multiplication by matrix units, since by Corollary 1.1 we can always find a basis which respects the deformation of a Wedderburn set. Thus the problem of classifying deformations is reduced to the problem of classifying deformations of sober algebras.

DEFINITION. A property $(P)$ of finite dimensional algebras over $K$ is open if whenever $(P)$ holds for $B_{0}$, then for every deformation $B$ of $B_{0}$ over an algebraic scheme $\left(C, t_{0}\right)$, it holds for all the closed fibers in a Zariski open neighborhood of $t_{0}$.

DEFINITION. We will say that a property $(P)$ is preserved by Morita equivalence if $(P)$ holds for $A$ if and only if it holds for the skeleton $A^{-}$of $A$.

COROLlaRY 1.3. If $(P)$ is a property preserved by Morita equivalence, then it suffices to check the openness of $(P)$ on deformations of sober algebras.

Proof. Let $B_{0}$ be any algebra, $B$ any deformation. We find $p:\left(\mathscr{C}^{\prime}, t_{0}^{\prime}\right) \rightarrow$ $\left(\mathscr{C}, t_{0}\right)$ as in Corollary 1.2 such that the fibers of $B^{\prime} \times \mathscr{C} \mathscr{C}^{\prime}$ are Morita equivalent to fibers of a deformation $\bar{B}^{\prime}$ of $B_{0}^{-}$. Let $U$ be an open neighborhood of $t_{0}^{\prime}$ over which the property $(P)$ holds in $\bar{B}^{\prime}$. Since $(P)$ is preserved by Morita equivalence, $(P)$ hold for all the closed fibers over $U$ in $B^{\prime}$. However, these are identical with the closed fibers over $p(U)$ in $\mathscr{C} . p(U)$ is the image of a morphism of finite type, and thus constructible. Since $p$ is etale over an algebraically closed field, it induces an isomorphism on the completions at $t_{0}$ and $t_{0}^{\prime}$ and thus $p(U)$ is actually a neighborhood of $t_{0}$ as required. 
3. Applications to deformation theory. We wish now to apply the existence of lifting of idempotents to reduce the set of possible deformations or specializations of an algebra.

DEFInItion. (1) The basis-graph of an algebra $A$ is obtained in the following way:

(i) We take a set of vertices corresponding to primitive orthogonal idempotents $e_{0}, \ldots, e_{r}$ with $e_{0}+\cdots+e_{r}=1$.

(ii) Let $n_{i j}=\operatorname{dim} e_{i} A e_{j}$. Each pair of distinct idempotents $e_{i}$ and $e_{j}$ will be connected by $n_{i j}$ arrows with a barb pointing from $e_{j}$ to $e_{i}$, and we add $n_{i i}-1$ loops at $e_{i}$.

(2) Let $J=\operatorname{Rad} A$, and let $n_{i j}^{k}=\operatorname{dim} e_{i}\left(J^{k} / J^{k+1}\right) e_{j}$. We will construct the weighted basis-graph of $A$ by putting $k$ barbs on $n_{i j}^{k}$ arrows or loops for each $k=$ $1,2, \ldots$ If $e_{i} A e_{j}$ contains a matrix unit, it will be represented by a solid triangular barb.

REMARK. Since any other set of primitive orthogonal idempotents can be obtained from this by conjugating by a unit, the basis graph and weighted basis graph do not depend on the choice of idempotents.

REMARK. We are deliberately trying to distinguish our diagrams from the quiver of the algebra, which includes only the arrows of weight 1 . To obtain the quiver one first replaces the algebra by its skeleton, as defined in $\S 2$, and then eliminate all arrows of weight greater than 1 .

EXAMPLE. The first weighted basis graph in Figure 1 represents $M_{2}(K)$, and the second represents the upper triangular $3 \times 3$ matrices.
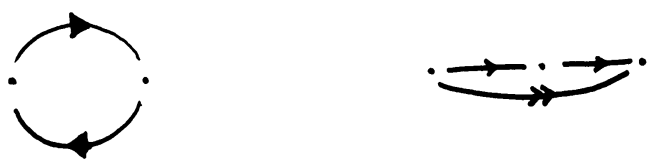

\section{FIGURE 1}

LEMMA 1. If $\bar{B}$ is a deformation over a nonsingular curve $\left(\mathscr{C}, t_{0}\right)$, then there is a flat family of ideals $\bar{J}$ such that $J_{t} \subseteq \operatorname{Rad} B_{t}$ everywhere, with equality holding almost everywhere.

PROOF. As is well known, $\operatorname{dim} \operatorname{Rad}\left(B_{t}\right)$ is an upper semicontinuous function of $t$, and thus this dimension equals some fixed minimal value $d$ on a Zariski open subset $U$ of $\mathscr{C}$. Let $\mathscr{G}_{r_{d, n-d}}$ be the Grassmann variety of $d$-dimensional subspaces of $V$. The conditions for being a two-sided nilpotent ideal are algebraic conditions depending on the structure constant tensor of $\bar{B}$, and therefore define an algebraic subvariety $Y$ of $\mathscr{C} \times \mathscr{G}_{r, n-d}$. Over $U$, where there is a unique ideal satisfying these conditions, we get a section $\sigma: U \times \mathscr{G}_{r_{d, n-d}}$. Let $W=\sigma(U)$. Since $\mathscr{G}_{r_{d, n-d}}$ is complete, the intersection of $\bar{W}$ with the fiber over $t_{0}$ is nonempty [8, II, 4.9, and Definition, p. 100].

However, in order to get a flat family of ideals, we need more than just the existence of such a point. The first projection of $W$ onto $U$ is an isomorphism, hence $\pi_{1}: \bar{W} \rightarrow \mathscr{C}$ is birational. A birational morphism onto a normal curve is an isomorphism so $\bar{W} \sim \mathscr{C}\left[\right.$ 8, V.3.8.1]. Thus we have a section $\mathscr{C} \rightarrow \mathscr{C} \times \mathscr{G}_{r_{d, n-d}}$ with 
image $\bar{W}$. Restricting to a Zariski neighborhood of $t_{0}$, this section determines a set of $R$-linearly independent elements of $B$, and we let $\bar{J}$ be the $R$-module generated by these elements. This family is flat at $t_{0}$, since it is free in a neighborhood of $t_{0}$. $J$ is a two-sided nilpotent subideal of $B$, since $W$ was a component in the variety of two-sided nilpotent ideals over $\mathscr{C}$. Since it is nilpotent, it is a subideal of $\operatorname{Rad} B$.

PROPOSITION 2. Let $\bar{B}$ be a deformation of $B$ over a curve $\left(\mathscr{C}, t_{0}\right)$, such that the dimension of the radical is constant in each fiber. Then the basis-graph is also constant and the function

$$
H_{i j}^{k}=\operatorname{dim} e_{i}\left(J / J^{k+1}\right) e_{j}
$$

giving the number of arrows with no more than $k$ barbs is upper semicontinuous.

PROOF. We first make a base extension, replacing $\mathscr{C}$ by its normalization, which will be a smooth curve. Flatness is preserved by base extension [8, III, 9.1A(b)]. Let $e$ be a complete primitive matrix decomposition set, and $x$ a basis which respects it, and which respects the family $\bar{J}$ of ideals guaranteed by Lemma 1 . By Corollary 1.1 we can find an etale cover $\mathscr{C}^{\prime}$ of $\mathscr{C}$ and sections $\tilde{e}, \tilde{x}$ such that $\tilde{x}$ respects $\tilde{e}$ and $J^{\prime}=\bar{J} \times \mathscr{C}^{\prime}$.

Let $s_{0}$ be the distinguished point of the new deformation $B^{\prime}=\bar{B} \times \mathscr{E} \mathscr{C}^{\prime}$ over $\left(\mathscr{C}^{\prime}, s_{0}\right)$. By hypothesis, $J=\bar{J}\left(t_{0}\right)$ was the radical of $B$, so $J^{\prime}\left(s_{0}\right)$ will be the radical of $B^{\prime}\left(s_{0}\right) \stackrel{\sim}{\rightarrow} B$. We divide the entire deformation by $J^{\prime} . B^{\prime} / J^{\prime}$ has a basis given by the sections of the semisimple set $\tilde{e}_{i}(s)$ over each point $s$, and this is a trivial deformation of $B / J$. Thus we have a fixed set of vertices, corresponding to the original idempotents.

Since $\tilde{x}$ respects $\tilde{e}$, the dimension $\tilde{e}_{i}(s) B^{\prime}(s) \tilde{e}_{j}(s)$ is also fixed whenever $\tilde{x}$ is a basis. Thus the number of arrows from vertex to vertex is also fixed.

Since $\tilde{x}$ respects $J^{\prime}, \operatorname{dim} \tilde{e}_{i} J \tilde{e}_{j}$ is constant. Thus we need to show that $\operatorname{dim} \tilde{e}_{i}\left(J^{\prime}\right)^{k+1} \tilde{e}_{j}$ is lower semicontinuous. The set $\tilde{e}_{i}\left(J^{\prime}\right)^{k+1} \tilde{e}_{j}$ is generated by the admissible products $\tilde{x}_{i_{1}} \cdots \tilde{x}_{i_{k+1}}$ of basis elements. The locus on which the dimension is $\leq r$ is defined by the vanishing of $(r+1) \times(r+1)$ minors and is thus an algebraic subvariety. Thus $\operatorname{dim} \tilde{e}_{i}\left(J^{\prime}\right)^{k+1} \tilde{e}_{j}$ is lower semicontinuous. In particular, it is constant over an open dense subset of $\mathscr{C}^{\prime}$, so we get a generic weighted basisgraph defined by these numbers, and these functions $H_{i j}^{k}$ are upper semicontinuous whenever the radical dimension is constant.

DEFINITION. The basis graph of a closed fiber over the open set on which the radical dimension is minimal will be called the generic basis graph of the deformation.

Proposition 3. Let $\bar{B}$ be a deformation of $B$ over $\left(\mathscr{C}, t_{0}\right)$. Then the basisgraph of $B$ either equals the generic basis-graph of $\bar{B}$ or is obtained from it by coalescing vertices, replacing each vanishing vertex by a loop.

PROOF. As before, we first replace $\mathscr{C}$ by its normalization. Let $e$ be a complete primitive semisimple set for $B, \bar{J}$ the flat family of two-sided ideals such that $\bar{J}_{s}=\operatorname{Rad} \bar{B}_{s}$ at the general point $s$. By passing to a base extension $\mathscr{C}^{\prime}$, we can find algebraic sections $\tilde{x}$ respecting $\tilde{e}$ and $J^{\prime}=\bar{J} \times \mathscr{C} \mathscr{C}^{\prime}$.

Let $s_{1}$ be any point whose basis-graph is generic. Since $\tilde{e}_{0} \cdots \tilde{e}_{l}$ form a set of idempotents in $s_{1}$, we can find a set of primitive idempotents subordinate to this 
set:

$$
f_{01}+\cdots+f_{0 m_{0}}=\tilde{e}_{0}\left(s_{1}\right), \ldots, f_{l 1}+\cdots+f_{l m_{l}}=\tilde{e}_{l}\left(s_{1}\right) .
$$

We then have

$$
n_{i j}=\operatorname{dim} \tilde{e}_{i}\left(s_{1}\right) \bar{B}_{s_{1}} \tilde{e}_{j}\left(s_{1}\right)=\sum \operatorname{dim} f_{i k} \bar{B}_{s_{1}} f_{j k} .
$$

Each vertex $e_{i}$ is obtained by coalescing $m_{i}$ vertices $f_{i 1}, \ldots, f_{i m_{i}}$. If $i \neq j$, then the number of arrows from $i$ to $j$ is the sum total of all the arrows from $f_{i p}$ to $f_{j q}$ for all $p=1, \ldots, m_{i}, q=1, \ldots, m_{j}$. If $i=j$, then the generic basis-graph contributes a number of loops equal to

$$
\sum_{k \neq k^{\prime}} n_{(i k)\left(i k^{\prime}\right)}+\sum_{k}\left(n_{(i k)(i k)}-1\right)=\left(\sum_{k, k^{\prime}} n_{(i k)(i k)}\right)-m_{i}=n_{i i}-m_{i} .
$$

There are actually $n_{i i}-1$ loops at $e_{i}$. Thus whenever $m_{i}$ vertices coalesce to one, we add $m_{i}-1$ loops to make up for the lost dimensions.

EXAMPLE. In Figure 2 we give an example of the weighted basis-graph of $M_{2} \oplus$ $M_{2}$ deforming to $M_{2}\left(k[t] /\left(t^{2}\right)\right)$.
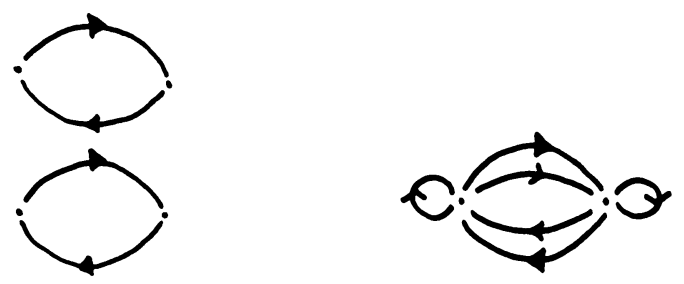

\section{FIGURE 2}

DEFINITION. Let $\operatorname{Alg}_{n}$ be the algebraic subscheme of $\operatorname{Spec}\left(K\left[c_{i j}^{k}\right]\right)$, the affine space containing all the structure constant tensors $\alpha=\left(a_{i j}^{k}\right)$ such that the multiplication

$$
v_{i} \cdot \alpha v_{j}=\sum a_{i j}^{k} v_{k}
$$

is associative and has an identity.

REMARK. This is the conventional parameter space for associative unitary $n$ dimensional algebras. The structure constant scheme $C_{n}$, as defined in Schaps [13], is the subscheme of $\mathrm{Alg}_{n}$ for which $v_{0}$ is the identity element.

DEFINITION. If $Q$ is a directed graph with $r$ vertices and $n-r$ arrows, then there is an $n$-dimensional radical-squared zero algebra, unique up to isomorphism, which has $Q$ as its basis graph and as its quiver. If we let $x_{1}, \ldots, x_{r}$ be the vertices and $x_{r+1}, \ldots, x_{n}$ be the arrows, the multiplication is given by

$$
\begin{array}{ll}
x_{i} \cdot x_{j}=\delta_{i j} x_{i} & i, j \leq r \\
x_{i} \cdot x_{k}=x_{k} & \text { if } x_{k} \text { denotes an arrow ending at vertex } x_{i}, \\
x_{k} \cdot x_{i}=x_{k} & \text { if } x_{k} \text { denotes an arrow beginning at vertex } x_{i}, \\
x_{k} \cdot x_{l}=0 & \text { if } k, l>r .
\end{array}
$$

We denote this algebra by $A_{Q}$ and call it the basis-graph algebra of $Q$. 


\section{Э}

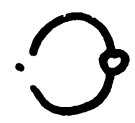

(i)

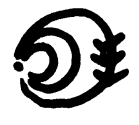

(ii)



(iii)

\section{FIGURE 3}

DEFINITION. We say that a directed graph $Q^{\prime}$ degenerates to a directed graph $Q$ if $Q$ is obtained from $Q^{\prime}$ by collapsing subsets of vertices and replacing each vanishing vertex with a loop, so that a subset of $m$ vertices is replaced by one vertex and $m-1$ loops.

THEOREM 1. Every irreducible component of $\mathrm{Alg}_{n}$ contains at most one loopless basis graph algebra, so the number of loopless directed graphs provides a lower bound to the number of irreducible components.

ProOF. Every irreducible component $W$ has a generic minimal radical dimension, and thus, by Proposition 2, has a generic basis-graph $Q^{\prime}$. If $A_{Q}$ is a basisgraph algebra with basis graph $Q$ in $W$, then $Q^{\prime}$ degenerates to $Q$ by Proposition 3. However, if idempotents in $Q^{\prime}$ coalesce to form $Q$, then $Q$ contains loops. If $Q$ is loopless, then $Q^{\prime}=Q$.

Propositions 2 and 3 give a fairly strong set of necessary conditions for the existence of deformations. For deformations over $k[[t]]$ these were already derived by Flanigan [3], although they do not seem to have passed into general use by representation theorists, perhaps because of the formal base space, or perhaps because they were not formulated in combinatorial terms.

What seems to have halted Flanigan's attack on the classification of finite dimensional algebras was the problem of giving sufficient conditions for the existence of deformations of what he calls type II, those involving creation of idempotents. We intend to remove this obstacle in a subsequent paper [2] by giving a diagonalization theorem for idempotent splitting deformations. This leads to an inductive procedure for constructing such deformations, inductive in that it depends on a knowledge of the deformation chart for $n-1$ dimensional algebras. We will also give a list of easily provable sufficiency conditions which in low dimensions will generate much of the chart in dimension $n$ from the chart in dimension $n-1$.

As an illustration of the weighted basis graph used in this section, we give a partial deformation chart in dimension 5, with the algebras placed as in Happel's chart in [7]. The lines indicate deformations implied by general sufficiency theorems. They are drawn solid when minimal, dotted otherwise. The only algebras included are those with a basis for which the product of two basis elements is another basis element times 0,1 , or -1 . Thus the continuous families are left out. 
Key to deformation charts (Figures 4 and 5). The symbols in Figure 3 represent the following:

(i) a loop whose products with every other radical element is zero;

(ii) the radical of $K[x] / x^{m+1}$, for $m=2$, and $m=3$;

(iii) the radical of $\Lambda^{2}(K)$, the alternating algebra on two generators.

4. Deforming algebras with distributive ideal lattice. It is known (see [12]) that every algebra of finite representation type has a distributive lattice of ideals. If we let $A_{j i}$ represent the Peirce component $e_{j} A e_{i}$, this means that $A_{j i}$ is a uniserial $\left(A_{j j}-A_{i i}\right)$-bimodule, i.e. has a unique chain of subbimodules. More specifically

(1) $A_{i i}$ is isomorphic to $E\left(m_{i}\right)=k[z] / z^{m_{i}+1}$.

(2) $A_{j i}$ is generated by a single element $y$ either as an $A_{j j}$-module or as an $A_{i i^{-}}$ module. That is, we have a basis $\left\{y, z_{j} y, \ldots, z_{j}^{d_{j}} y\right\}, d_{j} \leq m_{j}$, or $\left\{y, y z_{i}, \ldots, y z_{i}^{d_{i}}\right\}$, $d_{i} \leq m_{i}$.

The ideal lattice of an algebra is the same as the ideal lattice of its skeleton, so the property of having a distributive ideal lattice is preserved under Morita equivalence.

We now prove that the distributive ideal lattice property is an open condition, using the corollaries to Proposition 1 in $\S 2$. We remark that the definition of openness of a property $(P)$ in terms of deformations which is given in $\S 2$ is equivalent to saying that the set of closed points in $\mathrm{Alg}_{n}$ for which $(P)$ holds is the set of closed points in an open subset of $\mathrm{Alg}_{n}$. This is the definition used in P. Gabriel's proof of



FIGURE 4 


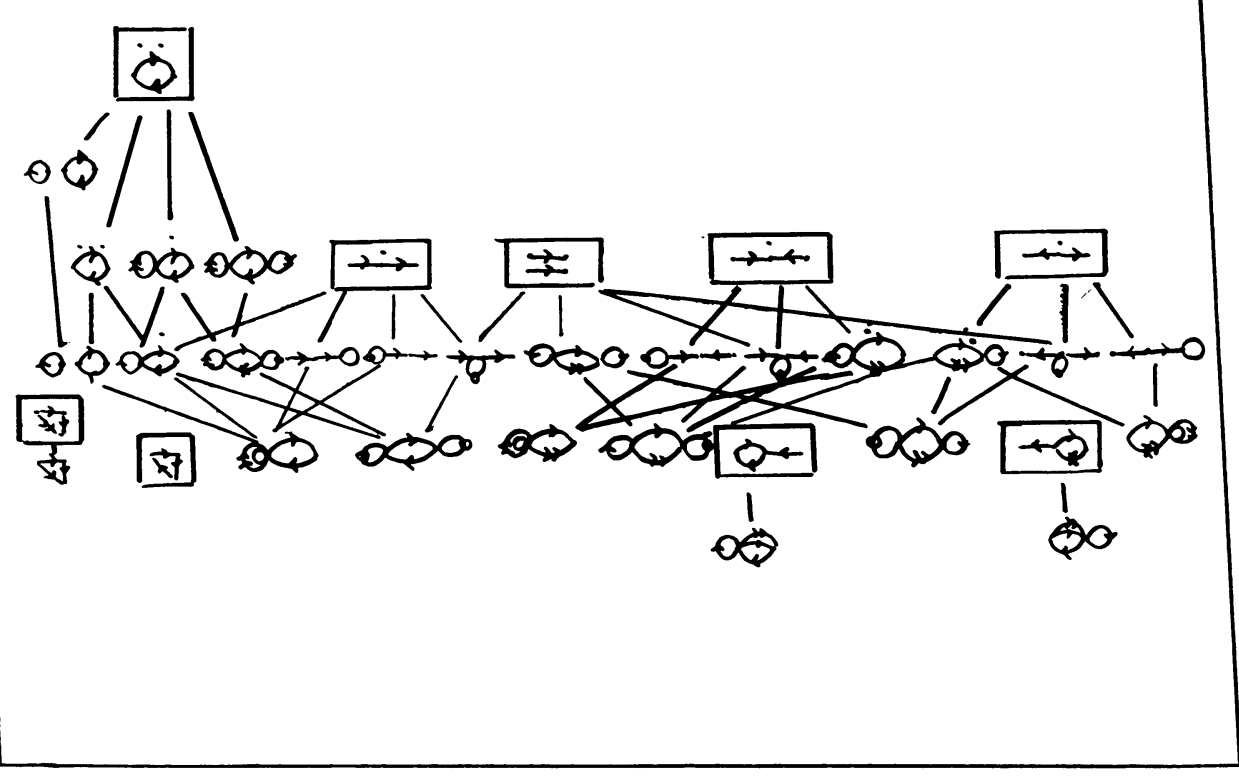

FigURE 5

the openness of finite representation type [5] and in A. Schofield's proof of openness of $t$-bounded global dimension [14].

PROPOSITION 4. Having a distributive ideal lattice is an open condition.

PROOF. Since the distributive ideal lattice condition is preserved under Morita equivalence, it suffices, by Corollary 1.3, to show that if $B_{0}$ is a sober algebra and $B$ is a deformation of $B_{0}$ over an algebraic scheme $\left(\mathscr{C}, t_{0}\right)$, then there is an open neighborhood of $t_{0}$ over which $(P)$ holds.

As we saw in the proof of Corollary 1.3 , replacing $\left(\mathscr{C}, t_{0}\right)$ by an etale neighborhood does not affect the test for openness, so we may assume that we have sections $f_{1}(t), \ldots, f_{r}(t)$ over $\mathscr{C}$ lifting the idempotents $f_{1}(0), \ldots, f_{r}(0)$ of $A_{0}$ and sections lifting elements of a basis $x_{0}$.

Let $A$ be the fiber over a general closed point $t$ of $\mathscr{C}$. We first show that each local Peirce block is a truncated polynomial ring. For each $i$, let $f_{i}^{\prime}$ be the idempotent in $A$ corresponding to $f_{i}(0)$ and let $z$ be the evaluation at $t$ of a section lifting the generator $z_{0}$ of the radical $f_{i}^{(0)} A_{0} f_{i}^{(0)} \tilde{\rightarrow} K[z] / z_{0}^{m+1}$. Since $f, z, z^{2}, \ldots, z^{m}$ are linearly independent in $A_{0}$, we must have $f, z, z^{2}, \ldots, z^{m}$ linearly independent in the general fiber $A$. Thus $f_{i}^{\prime} A_{0} f_{i}^{\prime}$ is commutative, of the form

$$
K[z] /\left(z^{m+1}+a_{m} z^{m}+\cdots+a_{0}\right), \quad a_{i} \in K .
$$

Since the field is algebraically closed, the polynomial factors in each closed fiber into a product of powers of linear factors, showing that $f_{i}^{\prime} A f_{i}^{\prime}$ is a product of truncated polynomial rings.

It remains to show that $f_{i} A f_{k}^{\prime} \neq 0$ is a uniserial $\left(f_{i} A f_{i}-f_{k}^{\prime} A f_{k}^{\prime}\right)$-bimodule for two primitive idempotents $f_{i}, f_{k}^{\prime}$ coming from decompositions of distinct idempotents $e_{0}, e_{0}^{\prime}$ in $B_{0}$. Let $e_{0}(t)$ be idempotent sections over $\mathscr{C}$, inducing idempotents 
$f_{0}, f_{0}^{\prime}$ in the fiber $A$, with $f_{0}=f_{1}+\cdots+f_{r}$, and $f_{0}^{\prime}=f_{1}^{\prime}+\cdots+f_{s}^{\prime}$. By the distributivity condition, $e_{0} B_{0} e_{0}^{\prime}$ is a uniserial $\left(e_{0} B_{0} e_{0}-e_{0}^{\prime} B_{0} e_{0}^{\prime}\right)$-bimodule. Without loss of generality we may assume that it is generated on the left by $e_{0} B_{0} e_{0} \tilde{\rightarrow} E(m)$. Let $y_{0}$ be the generator, and $z_{0}$ a generator of the radical of $e_{0} B e_{0}$. We then have

$$
e_{0} B_{0} e_{0}^{\prime}=\left\{y_{0}, z_{0} y_{0}, \ldots, z_{0}^{d} y_{0}\right\} .
$$

Let $\tilde{y}_{0}(t)$ be a section, and let $y \in A$ be the induced element of $A$. We let $z$ be an element of $A$ corresponding to $z_{0}$. We then have a basis $\left\{y, z y, \ldots, z^{d} y\right\}$ for $f_{0} A f_{0}^{\prime}$. This basis induces spanning sets for each of the Peirce components $f_{i} A f_{k}^{\prime}$. Letting $y_{i k}=f_{i} y f_{k}^{\prime}$, we conclude that $f_{i} A f_{k}^{\prime}$ is spanned by $\left\{y_{i k}, z_{i} y_{i k}, \ldots, z_{i}^{d} y_{i k}\right\}$ where $z_{i}=f_{i} z f_{i}$ is the $A_{i i}$-component of $z$. If $d^{\prime}=\operatorname{dim} f_{i} A f_{k}^{\prime}$, then there is some polynomial $h$ of minimal degree $d^{\prime \prime} \geq d^{\prime}$ such that $h\left(z_{i}\right) y_{i k}=0$. Every $z_{i}^{j} y_{i k}$ for $j \geq d^{\prime}$ can be expressed as a combination of terms of lower degree, so we conclude that $d^{\prime \prime}=d^{\prime}$, and $\left\{y_{i k}, \ldots, z^{d^{\prime}-1} y_{i k}\right\}$ is a basis. The proof is now complete. We mention, in conclusion, that since some power of $z_{i}$ is zero, some power of $z_{i}$ annihilates $y_{i k}$, and we can show inductively that in fact $h\left(z_{i}\right)=z_{i}^{d^{\prime}}$.

In Figure 5 we illustrate the deformation of algebras with distributive ideal lattice by giving the deformation chart for all such algebras whose basis-graph involves two or three arrows. Note that, by the first part of the proof of the theorem, two coalescing idempotents cannot be connected by an arrow. Therefore the number of arrows which are not loops in each connected component of the chart is fixed.

Appendix. We list here the various terms we will need from algebraic geometry. For simplicity, we have restricted ourselves to affine spaces.

DEFInITION. A morphism $f: \operatorname{Spec}(B) \rightarrow \operatorname{Spec}(A)$ is of finite type if the corresponding ring homomorphism $f: A \rightarrow B$ makes $B$ a finitely generated $A$-algebra. If $B$ is finitely generated not only as an algebra but even as a left $A$ module, then we say that $f$ is finite. (Geometrically this implies that there are only a finite number of points of $\operatorname{Spec} B$ over each point of $\operatorname{Spec}(A)$ [8, p. 84].)

DEFINITION. A morphism $f: Y \rightarrow X$ is etale if for every point $y \in Y$ and $x=f(y)$, there are affine neighborhoods $\operatorname{Spec}(B)$ of $y$ and $\operatorname{Spec}(A)$ of $x$ such that $B=A\left[t_{1}, \ldots, t_{n}\right] /\left(f_{1}, \ldots, f_{n}\right)$, with $\partial f / \partial t$ invertible $[\mathbf{1}$, p. 11].

NOTATION. Let $X$ be a scheme, and $x$ a point of $X$. Then $\mathscr{O}_{X, x}$ will denote the local ring of $X$ at $x$ in the Zariski topology. (If $\operatorname{Spec}(A)$ is an affine neighborhood of $x$, then $\mathscr{O}_{X, x}$ is the localization of $A$ at the prime ideal corresponding to $x$.) $\widehat{\mathscr{O}}_{X, x}$ will denote the completion of $\mathscr{O}_{X, x}$ at its unique maximal ideal, and $K(x)$ the residue field.

DEFINITION. Let $(X, x)$ be a pointed scheme. A pointed scheme $\left(X^{\prime}, x^{\prime}\right)$ together with an etale morphism $f: X^{\prime} \rightarrow X$ such that $f\left(x^{\prime}\right)=x$, and $K(x)=K\left(x^{\prime}\right)$ will be called an etale neighborhood of $(X, x)[\mathbf{1}$, p. 20].

REMARK. If $f: Y \rightarrow X$ is etale, and $f(y)=x$, then $\widehat{O}_{x, x} \otimes_{K(x)} K(y) \rightarrow \widehat{O}_{Y, y}$. If the ground field is algebraically closed, or $K(y)=K(x)$ as in an etale neighborhood, then $\widehat{O}_{X, x} \tilde{\rightarrow} \widehat{O}_{Y, y}$.

\section{BIBLIOGRAPHY}

1. M. Artin, Theoremes de representabilite pour les espaces algebriques, Les Presses de l'Universite de Montreal, 1973.

2. Th. Dana-Picard and M. Schaps, Classifying generic algebras, preprint, Bar-Ilan University. 
3. F. Flanigan, Straightening out and semi-rigidity in associative algebra, Trans. Amer. Math. Soc. 138 (1969), 415-425.

4. __ Which algebras deform into a total matrix algebra?, J. Algebra 29 (1974), 103-112.

5. P. Gabriel, Finite representation type is open, Representations of Algebras, Lecture Notes in Math., vol. 488, Springer-Verlag, 1974, pp. 132-155.

6. M. Gerstenhaber, On the deformation of rings and algebras, Ann. of Math. (2) 74 (1964).

7. D. Happel, Deformations of five dimensional algebras with unit, Ring Theory, Lecture Notes in Pure and Appl. Math., 1978.

8. R. Hartshorne, Algebraic geometry, Springer-Verlag, 1977.

9. N. Jacobson, Structure of rings, Amer. Math. Soc. Colloq. Publ., vol. 37, Amer. Math. Soc., Providence, R.I., 1964.

10. J. Lambek, Lectures on rings and modules, Blaisdell, Waltham, Mass., 1966.

11. G. Mazzola, The algebraic and geometric classification of associative algebras of dimension five, Manuscripta Math. 27 (1979), 81-101.

12. Ch. Reidtmann, Algebres de type de representation fini d'apres Bongartz, Gabriel, Roiter et d'autres, Seminaire Bourbaki, 37e annee, no. 650, 1985.

13. M. Schaps, Moduli of commutative and noncommutative covers, Israel J. Math. 58 (1987).

14. A. H. Schofield, Bounding the global dimension in terms of the dimension, Bull. London Math. Soc. 17 (1985), 393-394.

Department of MATHEMATICS, BAR-Ila.N UNIVERSity, RAMAT-GaN, ISRAEL 52100 